\title{
An Algorithm for Rigid-body Angular Velocity and Attitude Estimation Based on Isotropic Accelerometer Strapdowns
}

\author{
Ting Zou * \\ Postdoctoral Fellow \\ Department of Mechanical Engineering \\ and Centre for Intelligent Machines \\ McGill University, Montréal, Canada \\ Email: ting.zou@mail.mcgill.ca \\ Jorge Angeles \\ Professor, Fellow of ASME \\ Department of Mechanical Engineering \\ and Centre for Intelligent Machines \\ McGill University, Montréal, Canada \\ Email: angeles@cim.mcgill.ca
}

\begin{abstract}
A novel algorithm for the estimation of rigid-body angular velocity and attitude-the most challenging part of pose-and-twist estimation - based on isotropic accelerometer strapdowns, is proposed in this paper. Quaternions, which employ four parameters for attitude representation, provide a compact description without the drawbacks brought about by other representations, for example, the gimbal lock of Euler angles. Within the framework of quaternions for rigid-body angular velocity and attitude estimation, the proposed methodology automatically preserves the unit norm of the quaternion, thus improving the accuracy and efficiency of the estimation. By virtue of the inherent nature of isotropic accelerometer strapdowns, the centripetal acceleration is filtered out, leaving only its tangential counterpart, to be estimated and updated. Meanwhile, using the proposed integration algorithm, the angular velocity and the quaternion, which are dependent only on the tangential acceleration, are calculated and updated at appropriate sampled instants for high accuracy. This strategy, which brings about robustness, allows for
\end{abstract}

*Address all correspondence to this author. 
relatively large time-step sizes, low memory demands and low computational complexity. The proposed algorithm is tested by simulation examples of the angular velocity and attitude estimation of a free-rotating brick and the endeffector of an industrial robot. The simulation results showcase the algorithm with low errors, as estimated based on energy conservation, and high-order rate of convergence, as compared with other algorithms in the literature.

Keywords: quaternion integration, accelerometer strapdown, angular velocity, attitude, estimation

\section{Introduction}

Estimation of rigid-body twist (point-velocity and angular velocity) and pose (point position and attitude) is a recurrent need in modern applications, involving air- and spacecraft, driverless automobiles and robots. The estimation can be realized by means of conventional Inertial Measurement Units (IMU), which consist of at least three uniaxial accelerometers to measure point acceleration and three gyroscopes to measure the three components of angular velocity [1]. Gyroscopes are able to yield direct measurement of the angular velocity, but suffer from drawbacks of complex architectures, bulkiness, and high cost, besides drifting. Therefore, gyroscope-free IMU (GF-IMU) using micromachined accelerometers, which are also named accelerometer strapdowns, are becoming more attractive [2,3]. A conventional accelerometer strapdown is an accelerometer layout composed of uni-, bi- and triaxial accelerometers to yield readouts of angular and point acceleration $[4,5]$. From these readouts, the rigid-body (strapdown carrier, or simply carrier) pose and twist can be updated. By far, the most challenging aspects in this context are angular velocity and attitude, hence the motivation of the work reported here. Because of the applicability to arbitrary planar motion, relatively less complicated fabrication processes, and high measurement accuracy, accelerometer strapdowns with biaxial accelerometers offer attractive alternatives [6,7]. Among biaxial accelerometer designs, an innovative concept for biaxial accelerometers, termed simplicial ${ }^{1}$ architecture, was proposed by Cardou and Angeles [8]. Based on an improved Simplicial-Biaxial-Accelerometer (SBA) design, a novel concept of isotropic SBA strapdown was proposed by Zou and Angeles [9]. By virtue of its inherent isotropy, the SBA strapdown is able to decouple the tangential and centripetal components of the rigid-body acceleration field [10]. By this means, the rigid-body angular-acceleration estimation becomes independent of the angular velocity, thus overcoming a hurdle in current accelerometer strapdowns for angular-velocity and attitude estimation [11].

The determination of rigid-body angular velocity and attitude can be expressed in two Cartesian coordinate systems: body-fixed (local, or strapdown) frame and inertial (global) frame [12]. Direct readouts of accelerometer strapdowns are described in the carrier-fixed local frame. A transformation from the local frame to the inertial frame is required to describe the strapdown readouts in the inertial coordinate system. Amongst the most commonly used transformation descriptions are Euler angles, rotation matrices and unit quaternions [13, 14].

The three Euler angles describe the orientation of a rigid-body with respect to a predetermined fixed coordinate system [15]. Although simple to use, the well known gimbal-lock singularity, intrinsic to the Euler angles, limits their applications in rotation computations [16].

\footnotetext{
${ }^{1}$ Paper [8] title carries the wrong qualifier "simplectic." Ting Zou
} 
The $3 \times 3$ rotation matrix is also commonly used to describe the rigid-body attitude [17]. The orthogonal rotation matrix has nine components; however, they are not independent. The matrix column vectors are of unit Euclidean normintroducing three constraints — and orthogonal to each other-introducing three additional constraints. Hence, nine components and six constraints yield three independent scalars $[18,19]$. Enforcement of these constraints for complex systems in dynamic simulation is computationally expensive [20] and, may we add, unreliable.

Unit quaternions, an elegant, straightforward and computationally robust tool for rotation description, is attractive and widely used. Four parameters, namely, one scalar and one three-dimensional vector, make up the quaternion [21,22]. A unit quaternion is defined upon normalizing its components. The normalization is significant in the accuracy and computational efficiency for continuous tracking of the rigid-body angular velocity and attitude. In some integration algorithms in the literature, renormalization is required at each time step to keep the quaternion of unit norm. Errors may be incurred, due to the renormalization, and accumulated during the time span of integration [18,23].

Instead of renormalization, an integration algorithm that automatically preserves the quaternion unit norm was proposed [24]. The algorithm provides a closed-form solution of the quaternion integration, thereby yielding accurate results without the roundoff errors produced by classical integration algorithms; however, this algorithm is too complex, and hence, error-prone. Based on the above concept [24], the integration algorithm was improved by means of a predictor-corrector scheme that automatically preserves the unit norm during the updating of the rigid-body angular velocity and attitude estimation at each time step [25]. By doing this, renormalization is avoided, thus increasing computational efficiency and accuracy. Improvements on the predictor-corrector methodology were proposed to enforce the evaluation of position- and velocity-dependent force and torque constraints [20]. The predictor-corrector method predicts the approximate angular velocity at the next time step, by means of the quaternion at the current time step. The corrected angular velocity at the new time step is determined by the unit quaternion at the same time step via the quaternion multiplication rules.

With increasing demands for accuracy in the estimation algorithm, improvements on the quaternion integration technique become an effective means to improve the accuracy and robustness of the estimation. Many industrial systems have high degrees of freedom and multiple force and torque constraints. Within their long operation period, accurate quaternion integration with relatively large time steps under time-varying constraints is very attractive, but challenging.

In this paper the authors propose a novel estimation algorithm, based on an innovative isotropic accelerometer strapdown design, for the angular velocity and attitude estimation of a rigid body. Within this methodology, the quaternion at each time step is not obtained via a Taylor expansion, i.e., the addition or subtraction of quaternions at the previous time steps, but use direct quaternion-multiplication rules. Upon doing this, the quaternion norm is intrinsically preserved. By virtue of the isotropic accelerometer strapdown, the rotation matrix is directly obtained by the unit quaternion at the same time step. No approximation of the unit quaternion is required. Moreover, the isotropic accelerometer strapdown is able to decouple the tangential from the centripetal component of the angular acceleration under estimation, leaving only the tangential acceleration components to be estimated. This feature helps to greatly ease the estimation. Based on the isotropic accelerometer strapdown, the proposed estimation algorithm provides a straightforward approach to determining the angular acceleration, angular velocity and rigid-body attitude. No prediction and correction of the unit quaternion is needed. Noise is handled 
with the aid of the unscented Kalman filter; detailed investigation on the accuracy and efficiency of the algorithm is provided. The proposed algorithm and other algorithms in the literature are applied on the test simulation examples: a) a free-rotating brick in space and b) an industrial robot, then compared, to showcase the advantages of ours. Finally, conclusions are drawn.

\section{Angular Velocity and Attitude Estimation in an Inertial Frame}

Accurate transformation from the local frame of the rigid body under probing, the carrier, to the inertial frame is essential. For example, in order to yield the angular velocity and attitude information of the end effector of an industrial robot, an accelerometer strapdown can be directly attached to the end effector, to provide estimation signals in the end-effector frame. The strapdown moves together with the end-effector, its angular velocity and attitude estimation being in the local frame. In order to obtain the accurate and non-delayed angular velocity and attitude information in the inertial frame, an appropriate estimation algorithm is critical. However, it is noteworthy that this algorithm is not a stand-alone concept, but highly dependent on the strapdown design.

\section{An Isotropic Accelerometer Strapdown}

\subsection{Simplicial-Accelerometers}

Based on a fusion of concepts of parallel-kinematics machines and mathematical programming ${ }^{2}$, a novel concept of acceleromter design was proposed, namely, simplicial multi-axial accelerometers [8]. Depending on the acceleration components to be measured, these accelerometers can be uniaxial (SUA), biaxial (SBA), or triaxial (STA). In simplicialaccelerometer design, the proof-mass is suspended by $n+1$ limbs ( $n=1,2$, and 3 for uni-, bi- and triaxial, respectively). Measurement redundancy by one extra limb not only offers robustness against measurement noise, but also enhances the structure stiffness, particularly in the motion directions other than those of interest.

The SBA design, illustrated in Fig. 1, offers arbitrary translational motions of the proof-mass parallel to its plane, while providing a high stiffness along the out-of-plane directions. To realize the design objectives, the proof-mass is attached to three ПП-joints in the same plane as the proof-mass, at $120^{\circ}$ from one another. The ПП-joint is the serial array of two $\Pi$-joints. A П-joint, in turn, is essentially a parallelogram linkage [27]. A detailed investigation on the SBA design with ПП-joints is available [9]. The SBA is designed for fabrication using Microelectromechanical Systems (MEMS) technology, by means of single-crystal silicon, with a Young modulus $E=1.618 \times 10^{5} \mathrm{MPa}$, Poisson ratio $v=0.222$ and density $\rho=$ $2.33 \times 10^{-15} \mathrm{~kg} / \mu \mathrm{m}^{3}$.

\subsection{Accelerometer Strapdowns}

Figure 2 illustrates the geometry of an isotropic accelerometer strapdown, in a regular-tetrahedron array. By virtue of its planar nature, four SBAs are attached onto the tetrahedron strapdown, one on each face. The proof-mass center of mass (c.o.m.) coincides with the centroid of each face of the strapdown. However, the isotropic accelerometer strapdown is not

\footnotetext{
${ }^{2}$ In mathematical programming, a "simplex" is a hyper-polyhedron in a $n$-dimensional space with the minimum number of vertices, i.e., $n+1$ [26].
} 
limited to tetrahedral layouts, but applies to all isotropic polyhedral strapdowns, e.g., the other four Platonic solids, Buckyballs, and so on. Meanwhile, the structural simplicity of the tetrahedral strapdown uses a minimum number of accelerometers and eases its assembly. In comparison with traditional accelerometer strapdowns, which employ uniaxial accelerometers, the isotropic SBA strapdown offers identical estimation sensitivity along any directions in a plane, thus improving the estimation accuracy in any direction within the plane. This is realized by the intrinsic planar elastostatic isotropy of the SBA employed in the strapdown. The proof-mass plane in the SBA includes two principal axes of translational stiffness, at right angles, but otherwise of arbitrary orientations within the plane. The two principal stiffnesses in the plane, $K_{x}, K_{y}$, are identical, thereby leading to structural isotropy in the plane.

The position vector of the $i$ th SBA c.o.m., located at $P_{i}$ in the inertial frame $\mathcal{J}$, is denoted as $\mathbf{p}_{i}$, the centroid $C$ of the strapdown, of position vector $\mathbf{c}$ in $\mathcal{J}$, being given by

$$
\mathbf{c}=\frac{1}{n} \sum_{i=1}^{n} \mathbf{p}_{i}
$$

where $n$ is the number of SBAs for the accelerometer strapdown; for the tetrahedron strapdown, apparently, $n=4$.

The electrical circuit system etched on the SBA yields signals associated with the displacement of the proof-mass in its plane, in the local coordinate system $\mathcal{F}_{i}$ of the $i$ th SBA, with origin at $P_{i}$. Two additional coordinate frames are also introduced, the strapdown-fixed coordinate frame $\mathcal{S}$, and the inertial coordinate frame $\mathcal{J}$. One single SBA is not sufficient to provide a complete estimation of the acceleration of the body under probing. The relative acceleration of each SBA proof-mass c.o.m. with respect to $C$ is expressed as

$$
\ddot{\mathbf{p}}_{i}-\ddot{\mathbf{c}}=\left(\dot{\Omega}+\Omega^{2}\right)\left(\mathbf{p}_{i}-\mathbf{c}\right), \quad i=1, \ldots, n
$$

in the inertial frame J, $\Omega$ denoting the cross-product matrix of the angular velocity vector $\omega$, defined as [28]

$$
\Omega=\operatorname{CPM}(\omega)=\frac{\partial(\omega \times \mathbf{r})}{\partial \mathbf{r}}, \quad \forall \mathbf{r} \in \mathbf{R}^{3}
$$

Also, a three-dimensional vector $\pi_{i}$ for the $i$ th SBA is introduced:

$$
\pi_{i}=\mathbf{p}_{i}-\mathbf{c}, i=1, \ldots, n
$$


Hence, based on eqs. (2) and (4), we have

$$
\ddot{\pi}_{i} \equiv \ddot{\mathbf{p}}_{i}-\ddot{\mathbf{c}}=\left(\dot{\Omega}+\Omega^{2}\right) \pi_{i}, \quad i=1, \ldots, n
$$

The acceleration $\ddot{\pi}_{i}$ is decomposed into two orthogonal components, tangential $\ddot{\pi}_{i}^{\perp}$ and centripetal $\ddot{\pi}_{i}^{\|}$, namely,

$$
\ddot{\pi}_{i}^{\perp}=\dot{\Omega} \pi_{i}, \quad \ddot{\pi}_{i}^{\|}=\Omega^{2} \pi_{i}, \quad i=1, \ldots, n
$$

The acceleration readout reported by the $i$ th SBA at point $P_{i}$ lies in the plane of the SBA, i.e., in the $i$ th face of the tetrahedron. With reference to Fig. 2, the position vector $\pi_{i}$ is perpendicular to the $i$ th face of the polyhedron containing point $P_{i}$. This property, termed henceforth the normality property, holds for all isotropic strapdowns ${ }^{3}$. By virtue of the geometric isotropy of the tetrahedron as well as the structural isotropy of the SBA, only tangential acceleration readouts are sensed, while filtering out their centripetal counterparts. This property is significant in simplifying the rigid-body angular velocity and attitude estimation algorithm.

A direct readout of the $i$ th SBA yields the relative tangential acceleration of $P_{i}$ w.r.t. $C$ expressed in its local coordinate frame $\mathcal{F}_{i}$. In order to express the readout in the inertial coordinate frame $\mathcal{J}$, two coordinate transformations are needed, as given by their associated rotation matrices: $\mathbf{R}_{i}$, the transformation matrix from $\mathcal{F}_{i}$ to $\mathcal{S}$, and $\mathbf{Q}$, the proper orthogonal matrix defining the attitude of the strapdown, i.e., of the carrier, which takes vectors represented in $\mathcal{S}$ into their representation in $\mathcal{J}$, namely ${ }^{4}$,

$$
\mathcal{S}_{\ddot{\pi}_{i}}^{\perp}=\mathbf{R}_{i}^{\mathcal{F}_{i}} \ddot{\pi}_{i}^{\perp}, \quad \ddot{\pi}_{i}^{\perp}=\mathbf{Q}^{\mathcal{S}} \ddot{\pi}_{i}^{\perp},
$$

Further, the $3 \times n$ array $\Pi$ is defined as

$$
\Pi \equiv\left[\pi_{1}, \pi_{2}, \cdots, \pi_{n}\right]
$$

with similar definitions for $\dot{\Pi}$ and $\ddot{\Pi}$.

Based on the definitions of eqs. (7) and (8), for one strapdown containing $n$ SBAs, assembling all $n$ equations in eq. (6)

\footnotetext{
${ }^{3}$ In fact, a parallelepipedal array, a brick, which is not isotropic, is also endowed with this property, as illustrated with the example of Subsection 4.1. ${ }^{4}$ For simplicity, henceforth all vectors expressed in $\mathcal{J}$ carry no left superscript.
} 
yields,

$$
\ddot{\Pi}^{\perp}=\dot{\Omega} \Pi, \quad \ddot{\Pi}^{\perp}=\mathbf{Q}\left[\mathbf{R}_{1}^{\mathcal{F}_{1}} \ddot{\pi}_{1}^{\perp} \mathbf{R}_{2}^{\mathcal{F}_{2}} \ddot{\pi}_{2}^{\perp} \cdots \mathbf{R}_{n}^{\mathcal{F}_{n}} \ddot{\pi}_{n}^{\perp}\right]
$$

where $\Pi$ is expressed in the inertial coordinate frame $\mathcal{J}$, its relation to the strapdown-fixed coordinate frame ${ }^{\mathcal{S}} \Pi$ being

$$
\Pi=\mathbf{Q}\left({ }^{\mathcal{S}} \Pi\right)
$$

Post-multiplying both sides of the first equation of eqs. (9) by $\Pi^{T}$ yields

$$
\ddot{\Pi}^{\perp} \Pi^{T}=\dot{\Omega} \mathbf{R}, \quad \mathbf{R} \equiv \Pi \Pi^{T}
$$

Now, taking the axial vector ${ }^{5}$ of both sides of eq. (11) produces

$$
\operatorname{vect}\left(\ddot{\Pi}^{\perp} \Pi^{T}\right)=\frac{1}{2} \mathbf{J} \dot{\omega}
$$

where $\mathbf{J}$ is a symmetric, positive-definite matrix, given by [28]

$$
\mathbf{J} \equiv \operatorname{tr}(\mathbf{R}) 1-\mathbf{R}=\mathbf{Q}^{\mathfrak{S}} \mathbf{J}^{T}
$$

in which 1 denotes the $3 \times 3$ identity matrix. In fact, $\mathbf{J}$ can be regarded as the $3 \times 3$ inertia matrix of an array of unit masses collocated at $\left\{P_{i}\right\}_{1}^{n}$, with respect to $C$. Since $\mathbf{R}$ is body-pose dependent, $\mathbf{J}$ is also. In strapdown-fixed coordinates, ${ }^{\mathcal{S}} \mathbf{J}$, given below, is constant:

$$
{ }^{\mathcal{S}} \mathbf{J}=\operatorname{tr}\left({ }^{\mathcal{S}} \mathbf{R}\right) 1-{ }^{\mathcal{S}} \mathbf{R}, \mathbf{R}=\mathbf{Q}^{\mathcal{S}} \Pi\left({ }^{\mathcal{S}} \Pi\right)^{T} \mathbf{Q}^{T}=\mathbf{Q}^{\mathcal{S}} \mathbf{R} \mathbf{Q}^{T}
$$

\footnotetext{
${ }^{5}$ The axial vector of a $3 \times 3$ matrix $\mathbf{A}$ is defined as $\mathbf{a} \equiv \operatorname{vect}(\mathbf{A}) \equiv \partial(\mathbf{a} \times \mathbf{v}) / \mathbf{v} \forall \mathbf{v} \in \mathbf{R}^{3}$, a vector invariant of $\mathbf{A}$ [28].
} 
The angular acceleration for an accelerometer strapdown is obtained from eq. (12) as

$$
\dot{\omega}=2 \mathbf{J}^{-1} \operatorname{vect}\left[\ddot{\Pi}^{\perp}(\Pi)^{T}\right]
$$

For an isotropic tetrahedral strapdown, ${ }^{\mathcal{S}} \mathbf{J}=\left(a^{2} / 9\right) 1$, where $a$ is the side length of the tetrahedron. It is noteworthy that, for any isotropic strapdowns with $n$ vertices, ${ }^{\mathcal{S}} \mathbf{J}$ is independent of the transformation matrix; it is only related to the side $a$ of the strapdown itself [11].

For brevity, ${ }^{\mathcal{S}} \mathbf{J}$ can be expressed as ${ }^{\mathcal{S}} \mathbf{J}=\sigma^{2} 1$, where $\sigma^{2}$ represents a scalar factor. Therefore, eq. (13) becomes

$$
\mathbf{J}=\sigma^{2} \mathbf{Q} 1 \mathbf{Q}^{T}=\sigma^{2} \mathbf{Q} \mathbf{Q}^{T}=\sigma^{2} 1={ }^{\mathcal{S}} \mathbf{J}
$$

Hence, for an isotropic SBA strapdown, the rotation matrix $\mathbf{Q}$ is filtered out, which leads to $\mathbf{J}={ }^{\mathcal{S}} \mathbf{J}$, i.e., matrix $\mathbf{J}$ of eq. (13) is immutable under a change of frame.

Consequently, based on the isotropic strapdown property, the angular acceleration is calculated as

$$
\dot{\omega}=2^{\mathcal{S}} \mathbf{J}^{-1} \operatorname{vect}\left[\mathbf{Q}^{\mathcal{S}} \ddot{\Pi}^{\perp}\left({ }^{\mathcal{S}} \Pi\right)^{T} \mathbf{Q}^{T}\right]=\frac{2}{\sigma^{2}} \mathbf{Q v e c t}\left[{ }^{\mathcal{S}} \ddot{\Pi}^{\perp}\left({ }^{\mathcal{S}} \Pi\right)^{T}\right]
$$

By virtue of the identity vect $\left(\mathbf{a b}{ }^{T}\right) \equiv-(1 / 2) \mathbf{a} \times \mathbf{b}$, the vect $(\cdot)$ expression above can be $\operatorname{simplified}$ as vect $\left[{ }^{\mathcal{S}} \ddot{\Pi}^{\perp}\left({ }^{\mathcal{S}} \Pi\right)^{T}\right]=$ $(1 / 2)^{\mathcal{S}} \mathbf{P}^{\mathcal{S}} \ddot{\pi}^{\perp}$, where ${ }^{\mathcal{S}} \mathbf{P}=\left[{ }^{\mathcal{S}} \Pi_{1}{ }^{\mathcal{S}} \Pi_{2} \ldots{ }^{\mathcal{S}} \Pi_{n}\right]$ denotes a row array of $n 3 \times 3$ blocks ${ }^{\mathcal{S}} \Pi_{i}$ that map any three-dimensional vector $\mathbf{r}$ into ${ }^{\mathcal{S}} \pi_{i} \times \mathbf{r},{ }^{\mathcal{S}} \ddot{\pi}^{\perp}=\left[\left({ }^{\mathcal{S}} \ddot{\pi}_{1}^{\perp}\right)^{T}\left(\mathcal{S}_{\ddot{\pi}_{2}}^{\perp}\right)^{T} \ldots\left(\mathcal{S}_{\ddot{\pi}_{n}^{\perp}}\right)^{T}\right]^{T}$ being a $3 n$-dimensional vector.

Hence, $\dot{\omega}$ of eq. (17) can be expressed

$$
\dot{\omega}=\frac{1}{\sigma^{2}} \mathbf{Q}^{\mathcal{S}} \mathbf{P}^{\mathcal{S}} \ddot{\pi} \perp
$$

Remarkably, since ${ }^{\mathcal{S}} \mathbf{P}$ is constant, it need be computed only once, then stored for online use.

Although the isotropic accelerometer strapdown greatly eases the angular acceleration estimation, with reference to eq. (18), the estimation still requires the rotation matrix $\mathbf{Q}$ at each time step. $\mathbf{Q}$ can be expressed via its unit quaternion, 
isomorphic to the four-dimensional array $\eta$ of Euler-Rodrigues parameters, namely,

$$
\eta=\left[\begin{array}{l}
\mathbf{q} \\
q_{0}
\end{array}\right] \equiv\left[\begin{array}{c}
\sin (\phi / 2) \mathbf{e} \\
\cos (\phi / 2)
\end{array}\right]
$$

in which the rotation matrix $\mathbf{Q}$ is characterized by an axis of direction parallel to the unit vector $\mathbf{e}$, and an angle of rotation $\phi$ about the axis:

$$
\mathbf{Q}=\left(q_{0}^{2}-\mathbf{q}^{T} \mathbf{q}\right) 1+2 \mathbf{q} \mathbf{q}^{\mathrm{T}}+2 q_{0} \operatorname{CPM}(\mathbf{q})
$$

with CPM introduced in eq. (3).

The estimation of the angular velocity is not directly obtained from the accelerometer strapdown readouts, but from the integration of the angular acceleration. Classical integration methods, such as Simpson's rule, Runge-Kutta, or centraldifferences, will always bring truncation errors. Moreover, the estimation of rigid-body attitude and angular velocity are not independent; they obey a linear relation between the time-rate of change of the array $\eta$ and the angular velocity, namely [28]

$$
\dot{\eta}=\mathbf{H} \omega
$$

with the $4 \times 3$ matrix $\mathbf{H}$ defined as

$$
\mathbf{H}=\frac{1}{2}\left[\begin{array}{c}
q_{0} 1-\operatorname{CPM}(\mathbf{q}) \\
-\mathbf{q}^{T}
\end{array}\right]
$$

Substitution of eq. (22) into eq. (21) leads to

$$
\left[\begin{array}{c}
\dot{\mathbf{q}} \\
\dot{q_{0}}
\end{array}\right]=\frac{1}{2}\left[\begin{array}{cc}
\Omega(t) & \omega(t) \\
-\omega^{T}(t) & 0
\end{array}\right]\left[\begin{array}{c}
\mathbf{q} \\
q_{0}
\end{array}\right]
$$

Hence, the precise integration of the angular acceleration plays a significant role in improving the estimation of the angular velocity and the rigid-body attitude. Classical integration methods are based on Talyor expansion and require addition or subtraction operations for the quaternion derivatives at each time step. However, the unit norm of the quaternion cannot be 
preserved due to these operations. In order to preserve the unit norm when applying the foregoing methods, renormalization on the updated quaternion is required at each time step. Even if the renormalization does not require a high computational effort, it may introduce roundoff error, which, even if small, may contribute significantly to the buildup of drift in the attitude-estimation algorithm.

Instead of using Taylor expansion, the algorithm proposed here is based on quaternion multiplication, which preserves the norm of the quaternion intrinsically, thus avoiding renormalization at each time step [24]. Based on this methodology, an integration algorithm, of the predictor-corrector type, is proposed. Within the methods in the literature, the time-rateof change $\dot{\eta}$ of the quaternion is known. However, no direct relationship between the angular acceleration signal and the quaternion is included in the algorithms. Moreover, none of the algorithms available has discussed the applications in rigid-body angular velocity and attitude estimation by means of accelerometer strapdowns. A novel estimation algorithm of angular velocity and rigid-body attitude, based on the integration of the quaternion, is proposed in the steps below:

1. Predict the angular acceleration at the $(n+1 / 4)^{\text {th }}$ time step based on the unit quaternion.

If the angular velocity at the $n^{\text {th }}$ time step is labeled $\omega_{n}$, the angular velocity at a quarter of the next time step is expressed as

$$
\omega_{n+\frac{1}{4}}=\omega_{n}+\frac{1}{4} \dot{\omega}_{n} \Delta t
$$

where $\Delta t=t_{n+1}-t_{n}, \dot{\omega}_{n}$ being the angular acceleration at the $n^{\text {th }}$ time step.

The rotation matrix at the $n^{\text {th }}$ time step, based on the current value $\eta_{n}$, with reference to eq. (20), is

$$
\mathbf{Q}_{n}=\left(q_{n}^{2}-\mathbf{q}_{n}^{T} \mathbf{q}_{n}\right) 1+2 \mathbf{q}_{n} \mathbf{q}_{n}^{\mathrm{T}}+2 q_{n} \operatorname{CPM}\left(\mathbf{q}_{\mathrm{n}}\right)
$$

where, for the sake of simplicity, $q_{n}$ and $\mathbf{q}_{n}$ denote the update of $q_{0}$ and, correspondingly, of $\mathbf{q}$, at the $n^{\text {th }}$ time step.

Within the framework of the scheme proposed by Seelen et al. [20], "quaternion multiplication algorithm" refers to the direct multiplication of two quaternions, namely,

$$
\eta_{n+\frac{1}{4}}=\left[c\left(\frac{\left\|\omega_{n+\frac{1}{4}}\right\| \Delta t}{8}\right), s\left(\frac{\left\|\omega_{n+\frac{1}{4}}\right\| \Delta t}{8}\right) \frac{\omega_{n+\frac{1}{4}}}{\left\|\omega_{n+\frac{1}{4}}\right\|}\right]\left[\begin{array}{c}
\mathbf{q}_{n} \\
q_{0 n}
\end{array}\right]
$$

where $c(\cdot)$ and $s(\cdot)$ stand for $\cos (\cdot)$ and $\sin (\cdot)$, respectively.

Hence, the quaternion at the $(n+1 / 4)^{\text {th }}$ time step is determined with respect to $\eta_{n}$, separated by time $(1 / 4) \Delta t$. With 
$\eta_{n+\frac{1}{4}}$ known, the rotation matrix at a quarter of the next time step is obtained as

$$
\begin{aligned}
\mathbf{Q}_{n+\frac{1}{4}}= & \left(q_{n+\frac{1}{4}}^{2}-\left\|\mathbf{q}_{n+\frac{1}{4}}\right\|^{2}\right) 1+2 \mathbf{q}_{n+\frac{1}{4}} \mathbf{q}_{n+\frac{1}{4}}^{\mathrm{T}} \\
& +2 q_{n+\frac{1}{4}} \operatorname{CPM}\left(\mathbf{q}_{\mathrm{n}+\frac{1}{4}}\right)
\end{aligned}
$$

For isotropic accelerometer strapdowns, the angular acceleration at the $(n+1 / 4)^{\text {th }}$ time step is

$$
\dot{\omega}_{n+\frac{1}{4}}=\frac{1}{\sigma^{2}} \mathbf{Q}_{n+\frac{1}{4}}^{\mathcal{S}} \mathbf{P}^{\mathcal{S}} \ddot{\pi}^{\perp}
$$

2. Calculate the angular acceleration and the angular velocity at the mid-point $(n+1 / 2)^{\text {th }}$ time step.

Both angular velocities and quaternions are calculated at proper "fractional" time steps between the $n$ and the $(n+1)^{\text {st }}$ steps. The angular velocity, angular acceleration and quaternion at step $n+1 / 4$ serve as initial guesses for the angular velocity at the mid-point $(n+1 / 2)^{\text {th }}$ time step:

$$
\omega_{n+\frac{1}{2}}=\omega_{n+\frac{1}{4}}+\frac{1}{4} \dot{\omega}_{n+\frac{1}{4}} \Delta t
$$

The quaternion at the mid-point step is determined following the direct multiplication relationship:

$$
\eta_{n+\frac{1}{2}}=\left[c\left(\frac{\left\|\omega_{n+\frac{1}{2}}\right\| \Delta t}{8}\right), s\left(\frac{\left\|\omega_{n+\frac{1}{2}}\right\| \Delta t}{8}\right) \frac{\omega_{n+\frac{1}{2}}}{\left\|\omega_{n+\frac{1}{2}}\right\|}\right]\left[\begin{array}{c}
\mathbf{q}_{n+\frac{1}{4}} \\
q_{n+\frac{1}{4}}
\end{array}\right]
$$

The rotation matrix at the mid-point time step is calculated following an approach similar to that in eq. (27), namely,

$$
\begin{aligned}
\mathbf{Q}_{n+\frac{1}{2}}= & \left(q_{n+\frac{1}{2}}^{2}-\mathbf{q}_{n+\frac{1}{2}}^{T} \mathbf{q}_{n+\frac{1}{2}}\right) 1+2 \mathbf{q}_{n+\frac{1}{2}} \mathbf{q}_{n+\frac{1}{2}}^{\mathrm{T}} \\
& +2 q_{n+\frac{1}{2}} \operatorname{CPM}\left(\mathbf{q}_{\mathrm{n}+\frac{1}{2}}\right)
\end{aligned}
$$

In turn, the angular acceleration at the mid-point time step is calculated based on the rotation matrix:

$$
\dot{\omega}_{n+\frac{1}{2}}=\frac{1}{\sigma^{2}} \mathbf{Q}_{n+\frac{1}{2}}^{\mathcal{S}} \mathbf{P}^{\mathcal{S}} \ddot{\pi}^{\perp}
$$


3. Update the angular velocity and the angular acceleration at the next time step $n+1$ based on their counterparts at the mid-point time step.

The angular velocity at step $n+1$ is calculated as

$$
\omega_{n+1}=\omega_{n+\frac{1}{2}}+\dot{\omega}_{n+\frac{1}{2}} \Delta t
$$

The direct multiplication, following an approach similar to that of time step $n+1 / 2$, yields

$$
\eta_{n+1}=\left[c\left(\frac{\left\|\omega_{n+1}\right\| \Delta t}{4}\right), s\left(\frac{\left\|\omega_{n+1}\right\| \Delta t}{4}\right) \frac{\omega_{n+1}}{\left\|\omega_{n+1}\right\|}\right]\left[\begin{array}{l}
\mathbf{q}_{n+\frac{1}{2}} \\
q_{n+\frac{1}{2}}
\end{array}\right]
$$

Now the rotation matrix follows:

$$
\begin{aligned}
\mathbf{Q}_{n+1}= & \left(q_{n+1}^{2}-\mathbf{q}_{n+1}^{T} \mathbf{q}_{n+1}\right) 1+2 \mathbf{q}_{n+1} \mathbf{q}_{n+1}^{\mathrm{T}} \\
& +2 q_{n+1} \operatorname{CPM}\left(\mathbf{q}_{\mathrm{n}+1}\right)
\end{aligned}
$$

According to the estimation algorithm of the isotropic accelerometer strapdown, the angular acceleration at the $(n+1)^{\text {st }}$ time step can be obtained as

$$
\dot{\omega}_{n+1}=\frac{1}{\sigma^{2}} \mathbf{Q}_{n+1}{ }^{\mathcal{S}} \mathbf{P}^{\mathcal{S}} \ddot{\pi}^{\perp}
$$

\section{End.}

The proposed estimation algorithm provides a straightforward approach to determining the angular velocity and attitude of the rigid body using the unit quaternion multiplication at appropriate time intervals. Estimation results at the next time step are calculated based on the results at a quarter and at a half time step, thus allowing for relatively large time steps, while yielding accurate estimates. Accuracy and efficiency of the proposed estimation algorithm are discussed in Section 4. 


\subsection{Coping with Noise}

Due to unavoidable noise, the $i^{\text {th }}$ accelerometer readout ${ }^{\mathcal{S}} \hat{\pi}_{i}^{\perp}(t)$ is expressed as the sum of three mutually independent vectors: the actual value of the point-acceleration ${ }^{\mathcal{S}} \ddot{\pi}_{r, i}^{\perp}(t)$, the bias error ${ }^{\mathcal{S}} \delta \ddot{\pi}_{b, i}^{\perp}$ and the noise error ${ }^{\mathcal{S}} \delta \ddot{\pi}_{v, i}^{\perp}$, namely,

$$
\mathcal{S} \hat{\vec{\pi}}_{i}^{\perp}(t)={ }^{\mathcal{S}} \ddot{\pi}_{r, i}^{\perp}(t)+{ }^{\mathcal{S}} \delta \ddot{\pi}_{b, i}^{\perp}(t)+{ }^{\mathcal{S}} \delta \ddot{\pi}_{v, i}^{\perp}(t)
$$

The bias and noise errors are modelled as independent random variables following a Gaussian distribution with zero mean and isotropic variances $\sigma_{b, i}^{2} \mathbf{1}_{3 \times 3}$ and $\sigma_{v, i}^{2} \mathbf{1}_{3 \times 3}$ :

$$
\left[\begin{array}{c}
{ }^{\mathcal{S}} \delta \ddot{\pi}_{b, i}^{\perp} \\
\mathcal{S} \delta \ddot{\pi}_{v, i}^{\perp}
\end{array}\right] \sim \mathcal{N}\left\{\left[\begin{array}{l}
\mathbf{0}_{3} \\
\mathbf{0}_{3}
\end{array}\right],\left[\begin{array}{cc}
\sigma_{b, i}^{2} \mathbf{1}_{3 \times 3} & \mathbf{O}_{3 \times 3} \\
\mathbf{O}_{3 \times 3} & \sigma_{v, i}^{2} \mathbf{1}_{3 \times 3}
\end{array}\right]\right\}
$$

where $0_{n}$ denotes the $n$-dimensional zero vector, $1_{n \times n}$ the $n \times n$ identity matrix and $\mathbf{O}_{m \times n}$ the $m \times n$ zero matrix. It is noteworthy that the bias error is assumed constant throughout, noise error to be white and normally distributed.

The bias and noise errors for the $n$ accelerometers in an isotropic accelerometer strapdown are grouped in $3 n$-dimensional vector form as ${ }^{S} \delta \ddot{\pi}_{b}^{\perp}$ and ${ }^{S} \delta \ddot{\pi}_{v}^{\perp}$, respectively.

Therefore, eq. (37) for an isotropic accelerometer strapdown takes the form

$$
{ }^{\mathcal{S}} \hat{\pi}^{\perp}={ }^{\mathcal{S}} \ddot{\pi}^{\perp}+{ }^{\mathcal{S}} \delta \ddot{\pi}_{b}^{\perp}+{ }^{\mathcal{S}} \delta \ddot{\pi}_{v}^{\perp}
$$

\subsection{Error Propagation}

With reference to eq. (18), let $\mathbf{y}=\alpha \equiv \dot{\omega}$, the estimator $\hat{\mathbf{y}}$ of $\mathbf{y}$ being defined as

$$
\hat{\mathbf{y}} \equiv \hat{\alpha}=\frac{1}{\sigma^{2}} \mathbf{Q}^{\mathcal{S}} \mathbf{P}^{\mathcal{S}} \hat{\tilde{\pi}}^{\perp}
$$

Likewise, with reference to $\delta \hat{\tilde{\pi}}^{\perp}$ in eq. (39), $\hat{\mathbf{y}}$ is decomposed as

$$
\hat{\mathbf{y}}=\mathbf{y}+\delta \mathbf{y}_{b}+\delta \mathbf{y}_{v}
$$

where $\mathbf{y} \equiv\left(1 / \sigma^{2}\right) \mathbf{Q}^{\mathcal{S}} \mathbf{P}^{\mathcal{S}} \ddot{\pi}^{\perp}, \delta \mathbf{y}_{b} \equiv\left(1 / \sigma^{2}\right) \mathbf{Q}^{\mathcal{S}} \mathbf{P}^{\mathcal{\delta}} \delta \ddot{\pi}_{b}^{\perp}$, and $\delta \mathbf{y}_{v} \equiv\left(1 / \sigma^{2}\right) \mathbf{Q}^{\mathcal{S}} \mathbf{P}^{\mathcal{\delta}} \delta \ddot{\pi}_{v}^{\perp}$. 
Based on eq. (38), following a Gaussian distribution, $\delta \mathbf{y}_{b}$ and $\delta \mathbf{y}_{\mathbf{v}}$ are expressed as

$$
\left[\begin{array}{l}
\delta \mathbf{y}_{b} \\
\delta \mathbf{y}_{v}
\end{array}\right] \sim \mathcal{N}\left\{0_{6},\left[\begin{array}{cc}
\Sigma_{y, b}^{2} & \mathbf{O}_{3 \times 3} \\
\mathbf{O}_{3 \times 3} & \Sigma_{y, v}^{2}
\end{array}\right]\right\}
$$

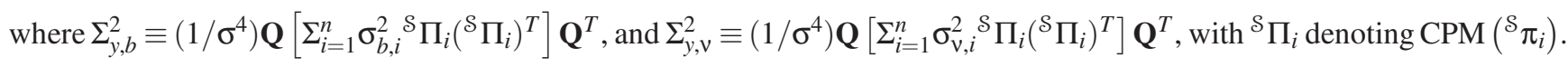

\subsection{State-space Model}

For the proposed estimation scheme, the state-space model is formulated as

$$
\begin{aligned}
\dot{\mathbf{x}} & =\mathbf{F} \mathbf{x}+\mathbf{G u} \\
\hat{\mathbf{y}} & =\mathbf{h}(\mathbf{x})+\delta \mathbf{y}_{\mathbf{v}}
\end{aligned}
$$

where

$$
\begin{aligned}
\mathbf{x} & =\left[\begin{array}{lll}
\alpha^{T} & \omega^{T} & \delta \mathbf{y}_{b}^{T}
\end{array}\right]^{T}, \delta \mathbf{y}_{b}=\delta \alpha_{b}, \mathbf{u}=\left[\begin{array}{ll}
\gamma^{T} & \beta_{\alpha}^{T}
\end{array}\right]^{T}, \\
\mathbf{F} & =\left[\begin{array}{ll}
\mathbf{O}_{3 \times 3} & \mathbf{O}_{3 \times 6} \\
1_{3 \times 3} & \mathbf{O}_{3 \times 6} \\
\mathbf{O}_{3 \times 3} & \mathbf{O}_{3 \times 6}
\end{array}\right], \mathbf{G}=\left[\begin{array}{ll}
1_{3 \times 3} & \mathbf{O}_{3 \times 3} \\
\mathbf{O}_{3 \times 3} & \mathbf{O}_{3 \times 3} \\
\mathbf{O}_{3 \times 3} & 1_{3 \times 3}
\end{array}\right], \mathbf{h}(\mathbf{x})=\alpha+\delta \alpha_{b}
\end{aligned}
$$

The system inputs $\left\{\gamma_{i}\right\}_{1}^{3}$ and $\left\{\beta_{\alpha, i}\right\}_{1}^{3}$ are the time-rates of change of the angular acceleration-termed the angular jerk - and those of the bias errors, respectively, which cannot be measured directly by an accelerometer strapdown. Hence, piecewise-constant functions are employed to model $\mathbf{u}_{k}$, a six-dimensional vector with random constant values, which follows a Gaussian distribution with zero mean and covariance matrix $\Sigma_{\mathbf{u}}^{2}$, namely,

$$
\mathbf{u}(t)=\left\{\begin{array}{lll}
\mathbf{u}_{0}, & \text { for } & t_{0} \leq t<t_{1} \\
\mathbf{u}_{1}, & \text { for } & t_{1} \leq t<t_{2} \\
\vdots & \vdots & \\
\mathbf{u}_{k}, & \text { for } & t_{k} \leq t<t_{k+1} \\
\vdots & \vdots &
\end{array} \quad \mathbf{u}_{k} \sim \mathcal{N}\left\{0_{6}, \Sigma_{\mathbf{u}}^{2}\right\}\right.
$$

The angular jerk and the time-rates of change of the bias errors are assumed to be stochastically independent, their 
covariance matrices taking the form:

$$
\Sigma_{\mathbf{u}}^{2}=\left[\begin{array}{cc}
\sigma_{\gamma}^{2} 1_{3 \times 3} & \mathbf{O}_{3 \times 3} \\
\mathbf{O}_{3 \times 3} & \sigma_{\beta}^{2} 1_{3 \times 3}
\end{array}\right]
$$

where $\sigma_{\gamma}^{2} 1_{3 \times 3}$ is the covariance matrix of $\gamma, \sigma_{\beta}^{2} 1_{3 \times 3}$ that of $\beta_{\alpha}$.

It is assumed that the initial estimate of the angular acceleration $\hat{\alpha}_{0}$ and that of the angular velocity $\hat{\omega}_{0}$ are independent, with Gaussian distributions of means $\alpha_{0}$ and $\omega_{0}$, respectively, and corresponding isotropic variances $\sigma_{\alpha, 0}^{2} 1_{3 \times 3}$ and $\sigma_{\omega, 0}^{2} 1_{3 \times 3}$. Therefore, the initial estimate $\hat{\mathbf{x}}_{0}$ for the state-space system above is given as

$$
\hat{\mathbf{x}}_{0} \equiv\left[\begin{array}{c}
\hat{\alpha}_{0} \\
\hat{\omega}_{0} \\
\delta \hat{\alpha}_{b}
\end{array}\right] \sim \mathcal{N}\left\{\left[\begin{array}{l}
\alpha_{0} \\
\omega_{0} \\
0_{3}
\end{array}\right],\left[\begin{array}{ccc}
\sigma_{\alpha, 0}^{2} 1_{3 \times 3} & \mathbf{O}_{3 \times 3} & \mathbf{O}_{3 \times 3} \\
\mathbf{O}_{3 \times 3} & \sigma_{\omega, 0}^{2} 1_{3 \times 3} & \mathbf{O}_{3 \times 3} \\
\mathbf{O}_{3 \times 3} & \mathbf{O}_{3 \times 3} & \Sigma_{y, b}^{2}
\end{array}\right]\right\}
$$

\subsection{Signal-to-noise Ratio}

The signal-to-noise ratio (SNR) is significant in the sensitivity analysis of accelerometer strapdowns, which is defined as [29]:

$$
S N R=20 \times \log _{10} \varepsilon
$$

where $\varepsilon$ denotes the error between the true angular acceleration vector $\alpha$ and the one contaminated with noise, $\hat{\alpha}$, namely,

$$
\varepsilon=\frac{\|\alpha\|_{2}}{\|\delta \alpha\|_{2}}=\frac{\|\alpha\|_{2}}{\|\alpha-\hat{\alpha}\|_{2}}
$$

with $\|\cdot\|_{2}$ representing the Euclidean norm of $(\cdot)$.

A highly obtrusive background noise will result in a low SNR, which means a low strapdown sensitivity.

\subsection{Unscented Kalman Filter}

In comparison with the commonly used extended Kalman filter (EKF), the unscented Kalman filter (UKF) [30-32] has superior implementation properties and performance. On the one hand, in the UKF, no Jacobian or Hessian calculations are required, which means a terser implementation. On the other hand, the fundamental idea underlying the UKF is the unscented transformation (UT), which is the method for calculating the statistics of a random variable undergoing a nonlinear 
transformation [31]. In the UT, a deterministic sampling procedure is conducted, in which the sampling points fully capture the true mean and covariance of the state variable, the a-posteriori mean and covariance thereby being accurate up to the second order for a nonlinear function. In contrast, the EKF can only achieve first-order accuracy. For details of the procedure to implement the UKF for coping with noise, the reader is referred to Appendix A.

\section{Simulation Results and Discussion}

The proposed estimation algorithm is tested with an example of the angular velocity and attitude estimation of a free rotating brick and the end-effector of an industrial robot. The estimation algorithm is tested and compared with the classical integration as well as the predictor-corrector method. Criteria for evaluating the comparison includes kinetic energy error, root-mean-square error, angular velocity, rate-of-convergence, and computational time.

\subsection{Free-rotating brick}

The brick, while not isotropic, is endowed with the normality property, introduced in Subsection 3.2, the property being exploited in this example.

Energy conservation is one of the most important criteria in evaluating the performance of numerical algorithms when integrating the governing equations [25]. The simulation example in testing the estimation algorithm based on energy conservation pertains to a free rotating brick in the absence of gravity, under conservative conditions. No external forces or moments are applied on the brick. Hence, the total mechanical energy is the kinetic energy of the brick, which remains constant, namely,

$$
E=\frac{1}{2} I_{x} \omega_{x}^{2}+\frac{1}{2} I_{y} \omega_{y}^{2}+\frac{1}{2} I_{z} \omega_{z}^{2}
$$

where $\mathbf{I}=\operatorname{diag}\left(I_{x}, I_{y}, I_{z}\right)$ is the inertia matrix of the brick, $\omega=\left[\omega_{x}, \omega_{y}, \omega_{z}\right]^{T}$ being its angular velocity.

The error in the energy conservation is expressed as

$$
\varepsilon=\left\|\frac{E_{n}-E_{0}}{E_{0}}\right\| \times 100 \%
$$

where $E_{n}$ is the kinetic energy at the $n^{\text {th }}$ integration time step, $E_{0}$ the initial kinetic energy.

One isotropic SBA strapdown is attached to the brick, as illustrated in Fig. 3. The dimensions of the free-rotating brick are given as $a=0.3 \mathrm{~m}, b=0.2 \mathrm{~m}$, and $c=0.1 \mathrm{~m}$. A frame $\mathcal{B}$ is attached to the brick under probing, with its origin $O$ located 
at the brick center of mass. The equation of motion of the brick is

$$
\mathbf{I} \dot{\omega}+\omega \times \mathbf{I} \omega=0_{3}, \quad \omega_{0}=[10,15,19]^{T} \mathrm{rad} / \mathrm{s}
$$

where $\mathbf{I}$ is the inertia matrix with respect to $O X Y Z$, expressed in frame $\mathcal{B}$, which is given as

$$
\mathbf{I}=\rho \operatorname{diag}(2.5,5.0,6.5) \times 10^{-3} \mathrm{~kg} \mathrm{~m}^{2}
$$

with a density $\rho=2700 \mathrm{~kg} / \mathrm{m}^{3}$. Assuming that $\omega_{0}$ is not parallel to any of the principal directions of inertia—coincident with the directions of the brick edges—-the brick will wobble.

Estimation algorithms based on three quaternion integration methods: a) classical Runge-Kutta; b) predictor-corrector; and c) the proposed algorithm, are investigated, then compared under energy-conservation conditions. The time-history of the angular velocity $\omega_{x}$ under estimation over a simulation interval of $2.0 \mathrm{~s}$ is shown in Fig. 4 . The error in the kinetic energy as a function of time is illustrated in Fig. 5. For all numerical methods, the time step size is set as $\Delta t=1.0 \times 10^{-4} \mathrm{~s}$.

The error in the total rotational energy for all numerical methods under test is small. However, the proposed method shows better energy conservation with smaller error. All methods show a slight increase of the error over simulation time, with the proposed method showing the smallest increase.

The computational time for different numerical methods is also recorded and compared. Over the simulation time of $2.0 \mathrm{~s}$, using the same time step size of $\Delta t=1.0 \times 10^{-4} \mathrm{~s}$, the computational time for the Runge-Kutta method, the predictorcorrector method and the proposed method are $0.875 \mathrm{~s}, 1.210 \mathrm{~s}$ and $0.944 \mathrm{~s}$, respectively.

Within the numerical integration of the proposed algorithm, the quaternion norm $\|\mathbf{q}\|$ is recorded at each time step, as illustrated in Fig. 6. It is apparent that the normality of the rotation quaternion is fairly well preserved.

For the free rotating brick, the SNR of the strapdown in estimating the angular acceleration is obtained as

$$
S N R=\left[\begin{array}{l}
S N R_{x} \\
S N R_{y} \\
S N R_{z}
\end{array}\right]=\left[\begin{array}{l}
35.68 \\
30.84 \\
30.12
\end{array}\right]
$$

\subsection{Industrial robot}

The second simulation example pertains to the angular velocity and attitude estimation of the end effector of an industrial robot, the PUMA 560. This is a typical six-axis robotic manipulator, developed by Victor Scheinman at Unimation.

The angular velocity and attitude of the PUMA 560 end-effector are estimated using the isotropic accelerometer strap- 
down, directly attached to the end-effector, as illustrated in Fig. 7. A Matlab-based toolbox to calculate the angular velocity and attitude of the end effector and joint motion of the robot between any two Cartesian poses, i.e., the initial and final poses, is available [33]. Figure 8 describes the spatial smooth path followed by the operation point of the end-effector (the end-effector tip) from the initial to the final position in three dimensional space, over the operational time of two seconds. End-effector orientation is illustrated at sampled points on the curve. The end-effector orientation is defined as that of the Frenet-Serret frame, a.k.a. the moving trihedron, composed of the tangent, normal and binormal unit vectors associated with every smooth curve [34]. This triplet of vectors, arranged, in order of listing, in a $3 \times 3$ matrix, represent the orientation of the smooth curve.

In robotics, the $4 \times 4$ homogeneous transformation matrix $\mathbf{T}$ is commonly used to represent the three-dimensional pose. The homogeneous matrix belongs to the special Euclidean group of dimension three, i.e., $\mathbf{T} \in \mathrm{SE}(3)$, which is expressed as

$$
\mathbf{T}=\left[\begin{array}{ll}
\mathbf{R} & \mathbf{p} \\
\mathbf{0}^{T} & 1
\end{array}\right]
$$

where 0 denotes three-dimensional zero vector, $\mathbf{R}$ the $3 \times 3$ rotation matrix, and $\mathbf{p}$ the position vector of the operation point of the end effector.

The $4 \times 4$ transformation matrix is used to represent the initial and final poses of the end-effector. The numerical values of this matrix, for the initial and the final poses, are

$$
\begin{aligned}
\mathbf{T}_{I} & =\left[\begin{array}{cccc}
1.000 & 0 & 0 & 0.400 \\
0 & -1.000 & 0 & 0.200 \\
0 & 0 & -1.000 & 0 \\
0 & 0 & 0 & 1.000
\end{array}\right] \\
\mathbf{T}_{F}= & {\left[\begin{array}{cccc}
0 & 0 & 1.000 & -0.400 \\
-1.000 & 0 & 0 & -0.200 \\
0 & -1.000 & 0 & 0.300 \\
0 & 0 & 0 & 1.000
\end{array}\right] }
\end{aligned}
$$

A smooth trajectory from the initial to the final pose is obtained by nonlinear interpolation, by means of 20,000 time steps over a simulation time of $2.0 \mathrm{~s}$. The angular velocity and attitude of the end-effector from the toolbox are recorded and compared with their counterparts by various estimation algorithms. Figure 9 illustrates the angular acceleration component $\dot{\omega}_{x}$ of the end-effector from the toolbox.

The estimation algorithms, including the quaternion integration by means of the classical Runge-Kutta method, the 
predictor-corrector method, and the proposed algorithm, are tested and compared. Figure 10 illustrates the estimation of the angular velocity component $\omega_{x}$ of the end-effector from various numerical methods, as a function of time over $2.0 \mathrm{~s}$ of simulation time. For all methods, the time step size is set as $\Delta t=1.0 \times 10^{-4} \mathrm{~s}$. All numerical methods show good agreement with the angular velocity from the toolbox output; apparently, the proposed algorithm shows the lowest error.

Using our algorithm, the time-histories of the quaternion components are plotted in Fig. 11. Within the proposed algorithm, the norm of the quaternion is automatically preserved, as shown in Fig. 12.

The rate of convergence for various numerical methods is investigated. The root-mean-square (rms) error with respect to the angular velocity $\omega_{x}$ from the toolbox output is calculated based on the error at each time step, namely,

$$
\beta=\frac{\Delta t}{T} \sum_{n=1}^{N} \beta_{n}
$$

where $\beta_{n}$ is the error at the $n^{\text {th }}$ time step, $N$ being the final time step over the simulation time $T=2 \mathrm{~s}$.

Due to the intrinsic preservation of the unit norm of the quaternion for both the predictor-corrector method and the proposed algorithm, the numerical integration of the unit quaternion will always converge.

The time step size is set from $\Delta t=1.0 \times 10^{-3} \mathrm{~s}$ to $\Delta t=1.0 \times 10^{-6} \mathrm{~s}$, for comparison of the rate of convergence, as the Runge-Kutta method does not converge for a larger time step. It is noteworthy from Fig. 13 that, for all the time step sizes in the test, all numerical methods converge; however, the predictor-corrector method and the proposed method show a superlinear rate of convergence, while the classical Runge-Kutta method shows a linear rate, apparent from the slope of the convergence curve.

For the industrial robot, the SNR of the strapdown in estimating the angular acceleration is obtained as

$$
S N R=\left[\begin{array}{l}
S N R_{x} \\
S N R_{y} \\
S N R_{z}
\end{array}\right]=\left[\begin{array}{l}
17.02 \\
22.78 \\
33.92
\end{array}\right]
$$

\section{Conclusions}

The authors proposed a novel algorithm for the estimation of rigid-body angular velocity and attitude using isotropic accelerometer strapdowns, based on unit-quaternion integration. The unit quaternion offers a simple, accurate description of rigid-body orientation. It has several intrinsic advantages over alternative representations like Euler angles and the full rotation matrix. On the one hand, the unit quaternion, which requires four parameters to specify the orientation, provides an accurate estimation without the gimbal-lock problem; on the other hand, Euler angles and rotation matrices suffer from inherent drawbacks of instability and singularity-occurrence. The unit-quaternion integration algorithm, based on the rules of quaternion multiplication, offers additional advantages, namely, improving accuracy and avoiding renormalization at each 
time step.

The isotropy of the accelerometer strapdown is capable of filtering out the centripetal acceleration component, leaving only the tangential component to be estimated, thus significantly easing the estimation procedure. By virtue of the isotropic strapdown, the proposed algorithm is able to provide a straightforward, efficient and accurate estimation of rigid-body angular velocity and attitude. A direct relationship between the angular acceleration and the quaternion is established, the proposed algorithm thus being simpler than the conventional predictor-corrector method. The angular acceleration, angular velocity and rigid-body attitude at time step $n+1$ are calculated with respect to a quarter $n+1 / 4$ and a half time step $n+1 / 2$. By doing this, the estimation algorithm allows for a relatively large time step, thus allowing for low memory demands while improving the computational efficiency. By virtue of intrinsic normalization of the quaternion, the proposed algorithm leads to a high accuracy. Noise is taken into account in the accelerometer readouts and handled by the unscented Kalman filter. The angular velocity and attitude estimation of a brick rotating freely in space and the end-effector of an industrial robot are used to test the proposed algorithm and its comparable counterparts. Simulation examples illustrate that energy conservation is met within a low error; the study on rate of convergence shows that the proposed algorithm has a superlinear convergence. As per the simulation results, the improved estimation algorithm for isotropic accelerometer strapdowns is accurate and efficient.

\section{Acknowledgements}

The second author would like to acknowledge the financial support received from NSERC (Canada's Natural Sciences and Engineering Research Council) through a Discovery Grant (No. 223213) and from McGill University via a James McGill Professorship (No. 100711).

\section{References}

[1] Yazdi, N., Ayazi, F., and Najafi, K., 1998. "Micromachined inertial sensors". Proceedings of the IEEE, 86(8), pp. 16401659.

[2] Mital, N. K., and King, A. I., 1979. "Computation of rigid-body rotation in three-dimensional space from body-fixed linear acceleration measurements". ASME J. Appl. Mech, 46(4), pp. 925-930.

[3] Pamadi, K. B., Ohlmeyer, E. J., and Pepitone, T. R., 2004. "Assessment of a GPS guided spinning projectile using an accelerometer-only IMU”. Proc. AIAA Guidance, Navigation, and Control Conference and Exhibit, pp. 705-717.

[4] Barbour, N., and Schmidt, G., 2001. "Inertial sensor technology trends". IEEE Sensors, 1(4), pp. 332-339.

[5] Cappa, P., Patanè, F., and Rossi, S., 2008. "Two calibration procedures for a gyroscope-free inertial measurement system based on a double-pendulum apparatus”. Meas. Sci. Technol., 19, pp. 32-38.

[6] Comi, C., Corigliano, A., Langfelder, G., Longoni, A., Tocchio, A., and Simon, B., 2011. "A new biaxial silicon resonant micro accelerometer". The Proceedings of the IEEE.

[7] Zou, Q., Tan, W., Kim, E., Singh, J., and E.Loeb, G., 2004. "Implantable biaxial piezoresistive accelerometer for sensorimotor control”. Proceedings of the 26th Annual International Conference of the IEEE EMBS. 
[8] Cardou, P., and Angeles, J., 2007. "Simplectic architectures for true multi-axial accelerometers: a novel application of parallel robots". IEEE International Conference on Robotics and Automation, pp. 181-186.

[9] Zou, T., and Angeles, J., 2014. "Structual and instrumentation design of a microelectromechanical systems biaxial accelerometer". Proc. IMechE Part C, J. Mechanical Engineering Science, 228(13), pp. 2440-2455.

[10] Zou, T., and Angeles, J., 2014. "The decoupling of the Cartesian stiffness matrix in the design of microaccelerometers". Multibody System Dynamics, 34(1), pp. 1-21.

[11] Zou, T., and Angeles, J., 2014. "Isotropic accelerometer strapdowns and related algorithms for rigid-body pose and twist estimation”. ASME J. Appl. Mech, 81(11), pp. 111001-1-111001-13.

[12] Spring, K. W., 1986. "Euler parameters and the use of quatenion algebra in the manipulation of finite rotations: a review". Mechanism and Machine Theory, 21(5), pp. 365-373.

[13] Terze, Z., Müller, A., and Zlatar, D., 2015. "Lie-group integration method for constrained multibody systems in state space”. Multibody Syst Dyn, 34, pp. 275-305.

[14] Treven, A., and Saje, M., 2015. "Integrating rotation and angular velocity from curvature". Advances in Engineering Software, 85, pp. 26-42.

[15] Kosenko, I., 1998. "Integration of the equations of a rotational motion of a rigid body in quaternion algebra, the Euler case”. J. Appl. Math. Mech., 62(2), pp. 193-200.

[16] Linne, M., 2002. Spectroscopic Measurement: An Introduction to the Fundamentals. Academic Press, San Diego.

[17] Eberly, D., 2002. Rotation representations and performance issues. Magic Software, Inc.

[18] Kuiper, J., 1999. Quaternions and Rotation Sequences. Princeton University Press, Princeton, US.

[19] Reich, S., and Zentrum, K., 1996. "Symplectic integrators for systems of rigid bodies". Integration Algorithms for Classical Mechanics, Fields Institute Commun., 10, pp. 181-191.

[20] Seelen, L., Padding, J., and Kuipers, J., 2016. "Improved quaternion-based integration scheme for rigid body motion". Acta Mechanica, pp. 1-9.

[21] Andrle, M., and Crassidis, J., 2013. "Geometric integration of quaternions". AIAA Journal of Guidance, Control, and Dynamics, 36(6), pp. 1762-1767.

[22] Zupan, E., and Saje, M., 2011. “Integrating rotation from angular velocity”. Advances in Engineering Software, 42, pp. 723-733.

[23] Betsch, P., and Siebert, R., 2009. "Rigid body dynamics in terms of quaternions: Hamiltonian formulation and conserving numerical integration”. Int. J. Numer. Methods Eng., 79(4), pp. 444-473.

[24] Whitmore, S., 2000. Closed-form integrator for the equaternion kinematics equations. Patent US6061611.

[25] Zhao, F., and van Wachem, B., 2013. "A novel quaternion integration approach for describing the behaviour of nonspherical particles". Acta Mechanica, 224, pp. 3091-3109.

[26] Kreyszig, E., 1997. Advanced Engineering Mathematics. John Wiley \& Sons, New York.

[27] Angeles, J., 2004. “The qualitative synthesis of parallel manipulators”. ASME J. Mechanical Design, 126(4), pp. 617624. 
[28] Angeles, J., 2014. Fundamentals of Robotic Mechanical Systems: Theory, Methods, and Algorithms. Fourth Edition, Springer, New York.

[29] Baltes, H., Brand, O., Fedder, G., Hierold, C., Korvink, J., and Tabata, O., 2005. Circuit and System Integration, Vol. 2. WILEY-VCH, Weinheim, Germany.

[30] Julier, S. J., Uhlmann, J. K., and Durrant-Whyte, H., 1995. "A new approach for filtering nonlinear systems". The Proceedings of the American Control Conference, pp. 1628-1632.

[31] Julier, S. J., and Uhlmann, J. K., 1997. "A new extension of the kalman filter to nonlinear systems". The Proc. of AeroSense: The 11th Int. Symp. on Aerospace/Defense Sensing, Simulation and Controls.

[32] Julier, S. J., and Uhlmann, J. K., 2004. “Unscented filtering and nonlinear estimation”. Vol. 46, The Proceedings of the IEEE, pp. 401-422.

[33] Corke, P. I., 2011. Robotics, Vision \& Control: Fundamental Algorithms in MATLAB. Springer. ISBN 978-3-64220143-1.

[34] Brand, L., 1965. Advanced Calculus. John Wiley \& Sons, New York.

[35] Brogan, W. L., 1991. Modern Control Theory. Prentice Hall.

\section{Appendix A: Working Process of the UKF}

\section{Selection of sigma points:}

The $n$-dimensional random variable $\mathbf{x}_{k-1}$ with mean $\hat{\mathbf{x}}_{k-1}$ and covariance $\mathbf{U}_{k-1}$ is approximated by a set $\chi_{k-1}$ of $2 n+1$ sigma points and their associated weights, given by

$$
\chi_{k-1}=\left\{\left(\mathbf{x}_{k-1}^{j}, W_{j}\right) \mid j=0 \ldots 2 n\right\}
$$

The sigma points are selected as explained below, which incorporates higher-order information:

$$
\begin{aligned}
& \mathbf{x}_{k-1}^{0}=\hat{\mathbf{x}}_{k-1} \\
& \mathbf{x}_{k-1}^{i}=\hat{\mathbf{x}}_{k-1}+\left(\sqrt{(n+\lambda)\left(\mathbf{U}_{k-1}\right)}\right)_{i}, i=1 \ldots, n \\
& \mathbf{x}_{k-1}^{i+n}=\hat{\mathbf{x}}_{k-1}-\left(\sqrt{(n+\lambda)\left(\mathbf{U}_{k-1}\right)}\right)_{i}, i=1 \ldots, n
\end{aligned}
$$

where $\lambda=\alpha^{2}(n+\kappa)-n$ is a scaling parameter, while $\alpha$ determines the spread of the sigma points around $\hat{\mathbf{x}}_{k-1}$, which is usually set to a small positive value, e.g., $10^{-4} \leq \alpha \leq 1$. As demonstrated elsewhere [32], the typical value of $\kappa$ is set to either 0 or $3-n$. Besides, $\left(\sqrt{(n+\lambda)\left(\mathbf{U}_{k-1}\right)}\right)$ refers to the $i^{\text {th }}$ column of the Cholesky factorization. 
The UKF includes two steps:

\section{(1) Model Forecast Step:}

The state-transition matrix is introduced for any time step $\Delta t=t_{k+1}-t_{k}$ :

$$
\Phi\left(t_{k}, t_{k+1}\right)=e^{\mathbf{F} \Delta t}=1_{n \times n}+\mathbf{F} \Delta t \equiv \Phi
$$

with the assumption that $\Delta t=h$ is constant. Then, according to Brogan [35], the system state at time $t_{k+1}$ is expressed as:

$$
\mathbf{x}_{k+1}=\Phi \mathbf{x}_{k}+\Upsilon \mathbf{u}_{k}
$$

where $\Upsilon=\mathbf{G} h+\mathbf{F G} h^{2} / 2$. Further, the discrete-time measurement equation takes a form similar to the continuous-time counterpart of eq. (43):

$$
\hat{\mathbf{y}}_{k}=\mathbf{h}\left(\mathbf{x}_{k}\right)+\delta \mathbf{y}_{v, k}
$$

Based on the UKF, each sigma point is propagated through the process model in eq. (59):

$$
\begin{aligned}
& \mathbf{x}_{k}^{f, i}=\mathbf{f}\left(\mathbf{x}_{k-1}^{i}\right)=\Phi \mathbf{x}_{k-1}^{i} \\
& \hat{\mathbf{x}}_{k}^{-}=\sum_{i=0}^{2 n} W_{i}^{m} \mathbf{x}_{k}^{f, i} \\
& \mathbf{V}_{k}^{-}=\sum_{i=0}^{2 n} W_{i}^{c}\left(\mathbf{x}_{k}^{f, i}-\hat{\mathbf{x}}_{k}^{-}\right)^{T}+\Upsilon \Sigma_{\mathbf{u}}^{2} \Upsilon^{T}
\end{aligned}
$$

with $W_{0}^{m}=\lambda /(n+\lambda), W_{0}^{c}=\lambda /(n+\lambda)+\left(1-\alpha^{2}+\beta\right), W_{i}^{m}=W_{i}^{c}=0.5 /(n+\lambda), i=1, \ldots, 2 n$, where $\beta$ is employed to incorporate the prior knowledge of the distribution of $x$.

Sigma points are propagated through the observation model of eq. (60) to obtain their predicted measurement counterparts:

$$
\mathbf{y}_{k}^{h, i}=\mathbf{h}\left(\mathbf{x}_{k-1}^{i}\right)
$$


The predicted observation $\hat{\mathbf{y}}_{k}^{-}$, the innovation covariance $\mathbf{V}_{\tilde{y}_{k} \tilde{y}_{k}}$ and the cross-correlation matrix $\mathbf{V}_{x_{k} y_{k}}$ are calculated as sample statistics of the sigma points:

$$
\begin{aligned}
\hat{\mathbf{y}}_{k}^{-} & =\sum_{i=0}^{2 n} W_{i}^{m} \mathbf{y}_{k}^{h, i} \\
\mathbf{V}_{\tilde{y}_{k} \tilde{y}_{k}} & =\sum_{i=0}^{2 n} W_{i}^{c}\left(\mathbf{y}_{k}^{h, i}-\hat{\mathbf{y}}_{k}^{-}\right)^{T}+\Sigma_{y, v}^{2} \\
\mathbf{V}_{x_{k} y_{k}} & =\sum_{i=0}^{2 n} W_{i}^{c}\left(\mathbf{x}_{k}^{h, i}-\hat{\mathbf{x}}_{k}^{-}\right)^{T}+\Sigma_{y, v}^{2}
\end{aligned}
$$

(2) Data Assimilation Step:

$$
\begin{aligned}
& \hat{\mathbf{x}}_{k}^{+}=\hat{\mathbf{x}}_{k}^{-}+\mathbf{K}_{k}\left(\mathbf{y}_{k}-\hat{\mathbf{y}}_{j}^{-}\right) \\
& \mathbf{K}_{k}=\mathbf{V}_{x_{k} y_{k}} \mathbf{V}_{\tilde{y}_{k} \tilde{y}_{k}}^{-1} \\
& \mathbf{V}_{k}^{+}=\mathbf{V}_{k}^{-}-\mathbf{K}_{k} \mathbf{V}_{\tilde{y}_{k} \tilde{y}_{k}} \mathbf{K}_{k}^{T}
\end{aligned}
$$




\section{List of Figures}

1 Simplicial Biaxial Accelerometer . . . . . . . . . . . . . . . . . . . 26

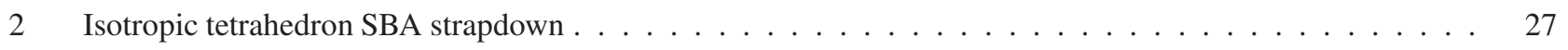

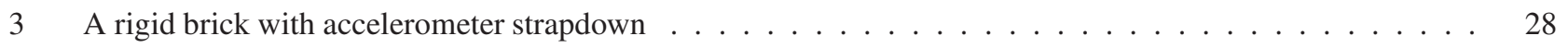

4 Comparison of the estimation of the angular-velocity time-history for three integration algorithms . . . . 29

5 Error in kinetic energy for three integration algorithms $\ldots \ldots \ldots$

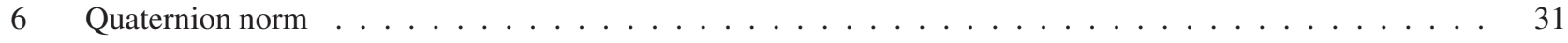

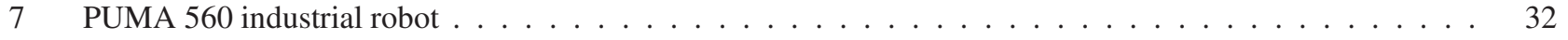

8 PUMA 560 operation point trajectory $\ldots \ldots \ldots \ldots$

9 PUMA 560 end-effector angular acceleration component $\dot{\omega}_{x} \ldots \ldots \ldots$

10 PUMA 560 end-effector estimated angular velocity component $\omega_{x} \ldots \ldots$

11 Quaternion components of the end-effector attitude . . . . . . . . . . . . . . . 36

12 Time-history of the computed norm of the quaternion . . . . . . . . . . . . . . 37

$13 \log -\log$ plot of error in $\omega_{x}$ vs the inverse of time step $1 / \Delta t \ldots \ldots \ldots$ 


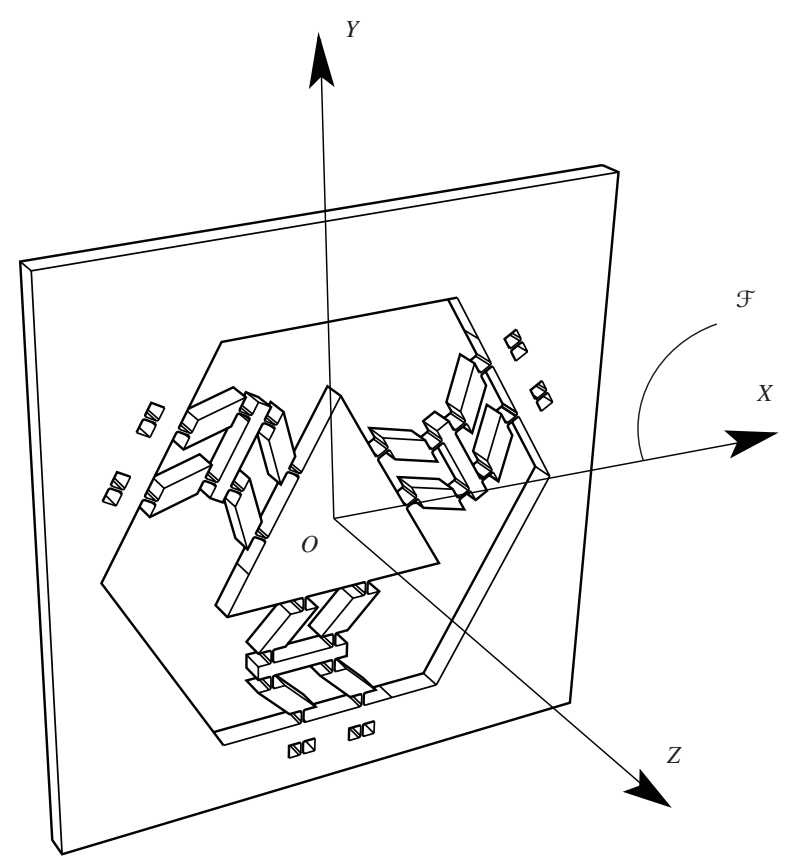

Fig. 1. Simplicial Biaxial Accelerometer 


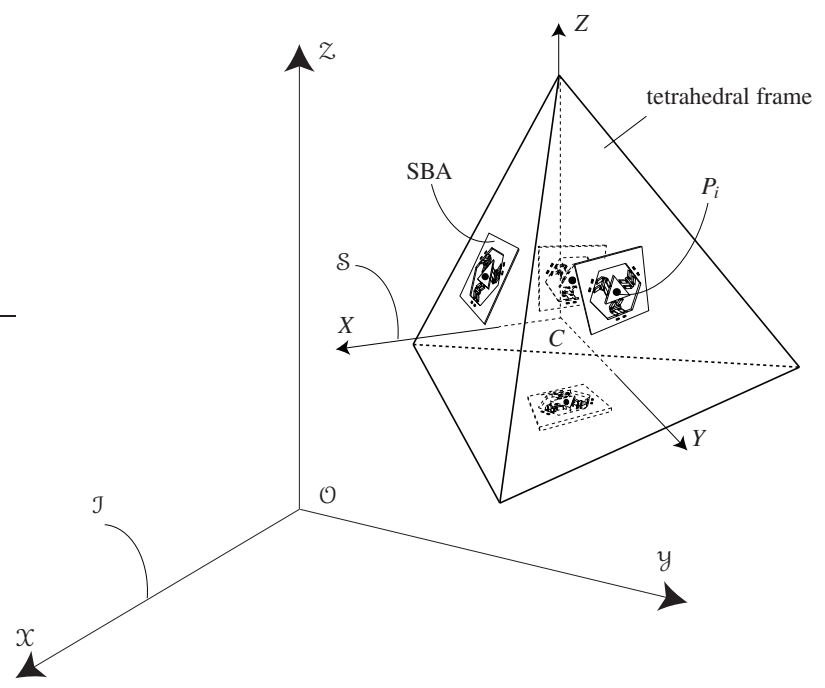

Fig. 2. Isotropic tetrahedron SBA strapdown 


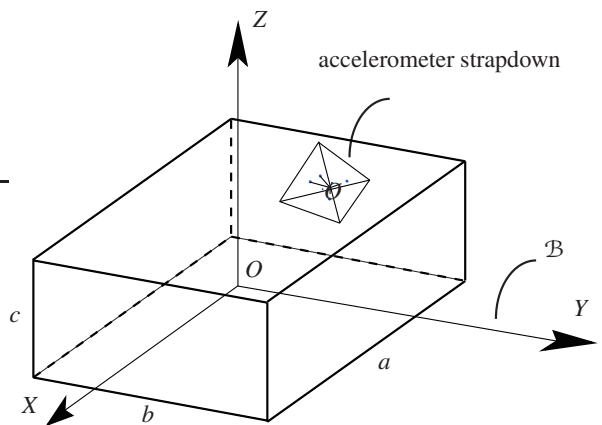

Fig. 3. A rigid brick with accelerometer strapdown 


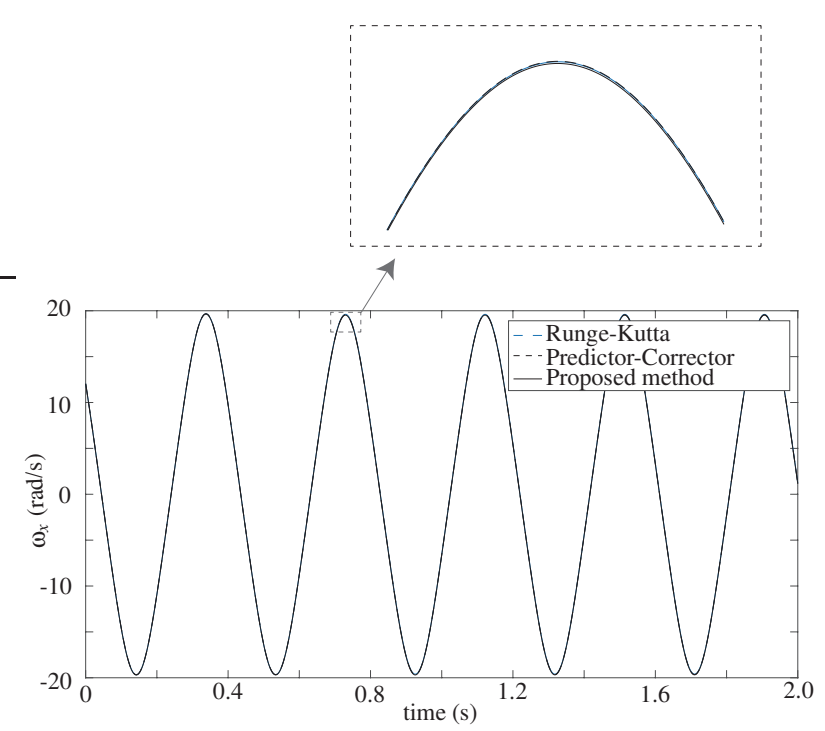

Fig. 4. Comparison of the estimation of the angular-velocity time-history for three integration algorithms 


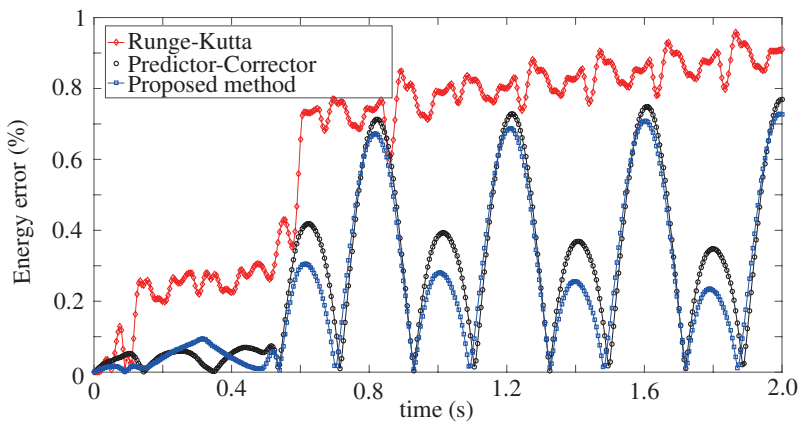

Fig. 5. Error in kinetic energy for three integration algorithms 


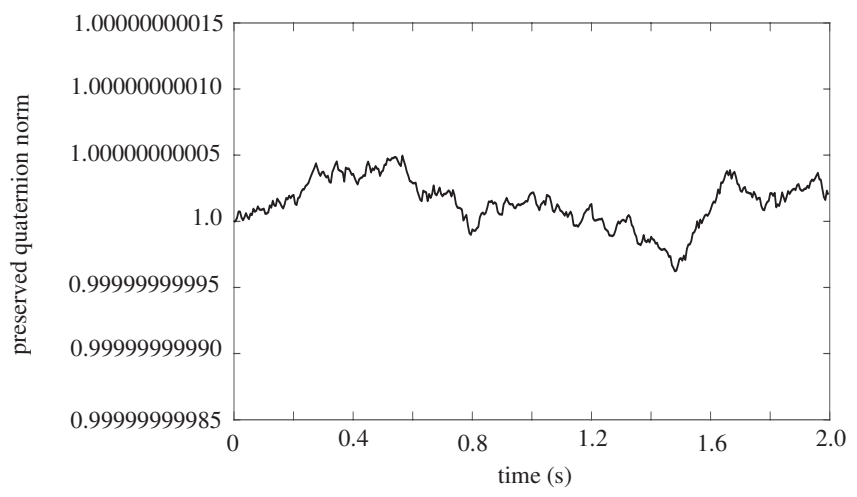

Fig. 6. Quaternion norm 


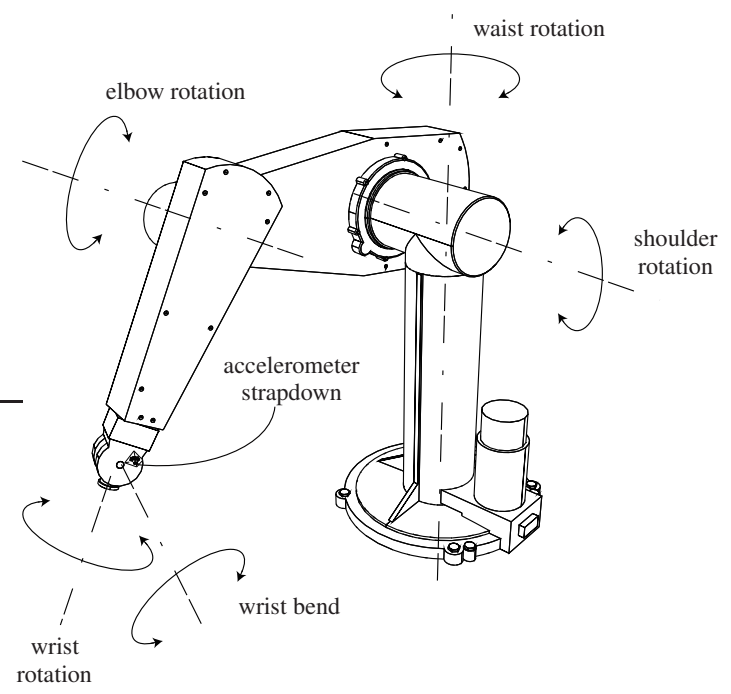

Fig. 7. PUMA 560 industrial robot 


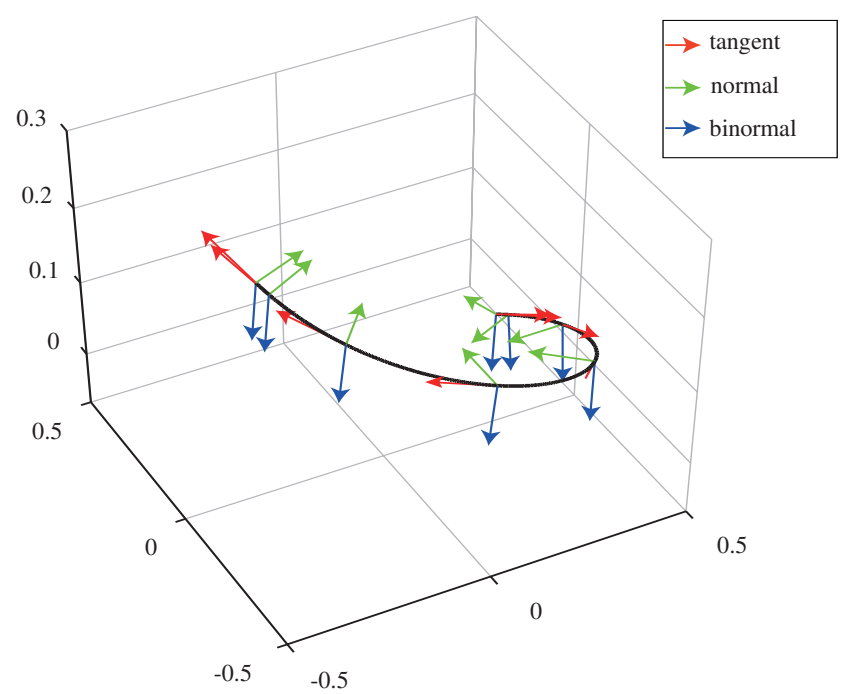

Fig. 8. PUMA 560 operation point trajectory 


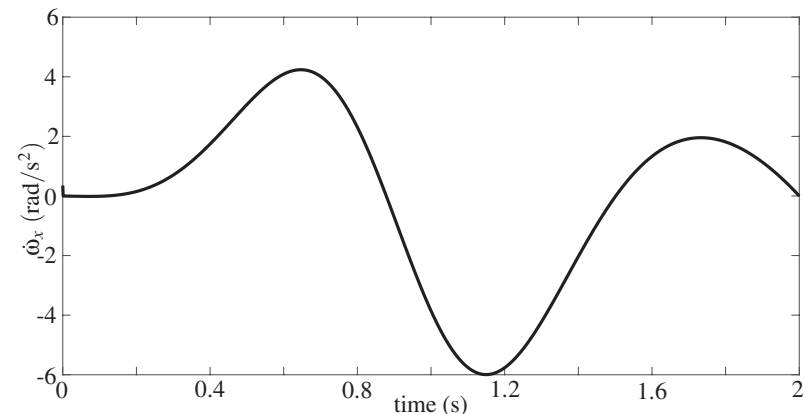

Fig. 9. PUMA 560 end-effector angular acceleration component $\dot{\omega}_{x}$ 


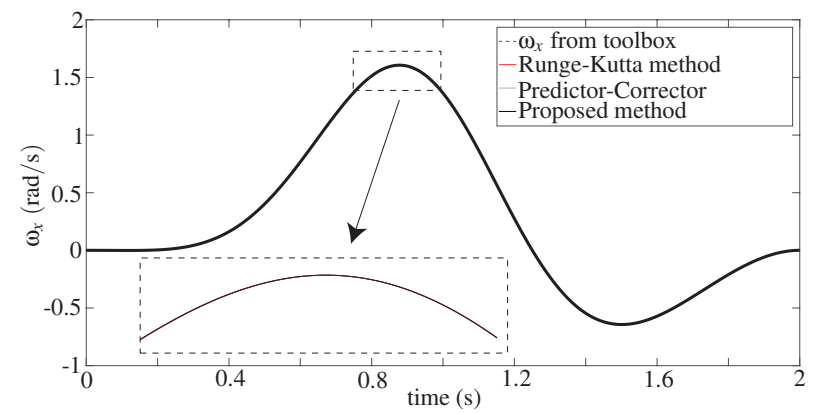

Fig. 10. PUMA 560 end-effector estimated angular velocity component $\omega_{x}$ 


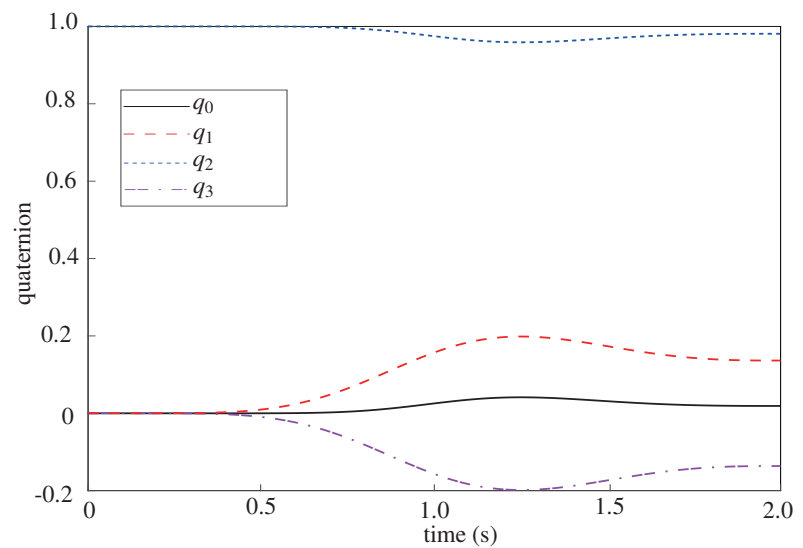

Fig. 11. Quaternion components of the end-effector attitude 


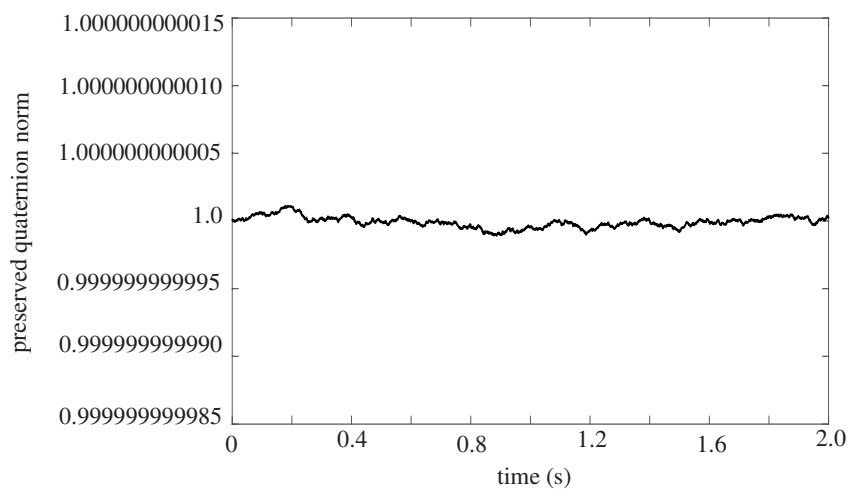

Fig. 12. Time-history of the computed norm of the quaternion 


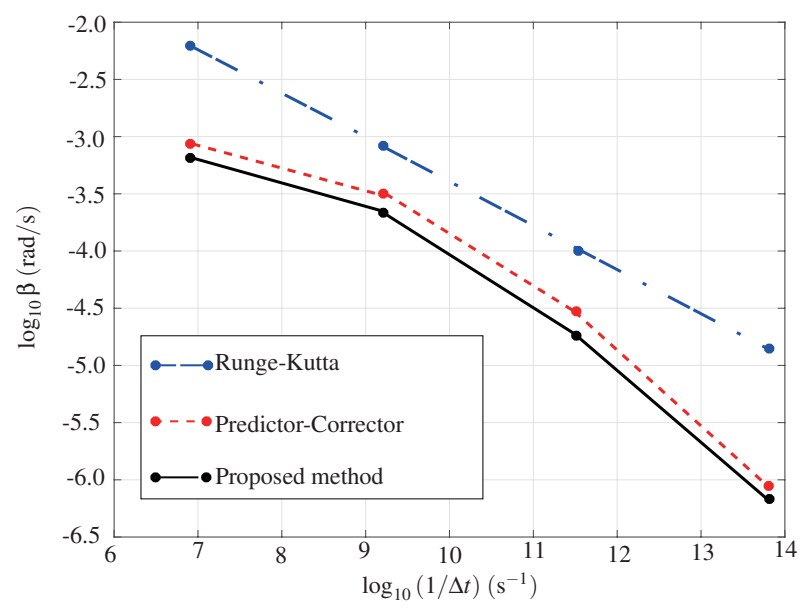

Fig. 13. $\log -\log$ plot of error in $\omega_{x}$ vs the inverse of time step $1 / \Delta t$ 